\title{
ENSEÑANZA DE LA LECTURA A PERSONAS ADULTAS CON TRASTORNOS DEL DESARROLLO
}

\author{
Hannia Cabezas Pizarro
}

\author{
Recibido 11-VI-2003 • Aceptado 12-VIII-2003
}

\begin{abstract}
Resumen: En este artículo se presenta un programa de intervención, para la adquisición de destrezas de lectura y escritura, en 8 mujeres adultas cuyas edades oscilan entre los 20 y los 48 años de edad, con retardo mental, y que no habían recibido escolaridad.

Se utiliza el modelo de destrezas propuesto por Skinner (1957), en el que hay una conducta observable que se quiere desarrollar, se le da seguimiento a través de un registro de eventos, y se grafican las destrezas obtenidas por cada una de las participantes, en el campo de la lectura, lo que permite ver el avance a través de las diferentes fases del proceso, además de ello, se aplicaron los modelos de investigación, en los que se pretende poner en relieve cambios de una serie de datos en una medida única o series de medidas. (Mayer y Labrador) 1980. Dentro de los modelos de investigación se utilizó el de criterio cambiante y múltiples $\mathrm{AB}$. En donde se investigan los efectos de una condición dada sobre una conducta específica. (Kazdin, 1980).
\end{abstract}

Palabras clave: Lectura, Problemas del Desarrollo, Adultos, Enseñanza, Alfabetización, Habilidades Lectoras, Métodos de Lectura.

\section{Antecedentes}

Se ha definido a las personas con necesidades educativas especiales, como aquellas que requieren condiciones de aprendizaje diferentes al resto de la población, por lo que las metodologías y los ajustes que realice el maestro, deben adecuarse a estas necesidades.

Los trastornos de inicio en la infancia, la niñez o la adolescencia, de acuerdo con el (DSM IV, 1997), son aquellos que "se presentan en la asistencia clínica durante la infancia o la adolescencia, a veces los trastornos en cuestión no se diagnostican hasta la etapa adulta". P. 39.

Hay variaciones que se atribuyen a etapas del desarrollo. El DSM IV 1997, por lo que "deben ser tenidas en cuenta las distintas etapas del desarrollo al evaluar las insuficiencias de las habilidades adaptativas, porque algunas de ellas son menos relevantes en edades distintas" p. 46 dentro de esta categoría se incluye el retado mental.

Las personas con retardo mental, necesitan de estrategias pedagógicas que tomen en cuenta sus habilidades, y que les ayuden a desarrollar al máximo su potencial.

El avance tecnológico de la vida moderna, ha aumentado las posibilidades de vida de las personas cuya esperanza en otra época se habría dificultado. Tal es el caso de los niños prematuros, de alto riesgo, o de aquellas personas con serias 
limitaciones físicas y mentales. Paralelamente, el nivel de vida de las personas con retado mental, también ha crecido, convirtiéndose en muchos casos en un problema social, porque el Estado no cuenta con los recursos necesarios para su atención.

Tomando en consideración los derechos de las personas con necesidades educativas especiales, el desarrollo integral se convierte en uno de ellos. Para esto, deben tomarse en consideración cuatro áreas fundamentales como son: El área educativa, el área recreativa, el área social y el área laboral, cuyo fin primordial será la integración al trabajo en la medida de sus posibilidades.

Al ser la educación pilar del desarrollo, y como parte de ella, la alfabetización, es por ello que esta investigación, giró en torno a un programa para la enseñanza de la lectura para personas adultas con problemas en el desarrollo, que no habían recibido escolaridad.

Educación, también es salud, si tomamos en consideración la necesidad del individuo de estar bien consigo mismo, de crecer en forma armónica y de sentirse útil a la sociedad, hablamos en este caso, de salud mental, por lo que este aspecto no debe abandonarse.

Tomando en consideración el derecho a la educación, se llevó a cabo un programa de lectura en el Taller ACOPANE, Institución dedicada a la atención de personas con retardo mental en el que participaron ocho muchachas quienes no habían recibido escolaridad, y en algunos casos no habían tenido ninguna oportunidad educativa.

\section{Fundamentos teóricos}

El ser humano no vive aislado, sino que existe una clara interdependencia entre él y las cosas, él y las personas, y el mundo que le rodea, por lo que sus actividades, se relacionan íntimamente con el resto de la comunidad.
El medio social, en donde se desenvuelve el ser humano, se convierte a su vez en un medio educativo, las vivencias ayudan a su crecimiento, y van desarrollando conductas que les permiten convivir con el resto de los ciudadanos, surgiendo una necesidad social de comunicación.

Dentro del medio social, se expone a los individuos a un proceso educativo, sistemático o no, que le permite una mejor convivencia. El desarrollo integral, no puede dejar de lado el aspecto académico. La educación es un derecho, y la necesidad de establecer programas sistemáticos que produzcan cambios de actitud, y la adquisición de destrezas, se hace necesaria para la población adulta con retardo mental. Estos programas, a su vez, le dan cabida a un mejor desempeño de acuerdo con sus habilidades.

El desarrollo en sí, no es solo el aprendizaje de costumbres e ideas, sino también contempla aquellos aspectos académicos mínimos, que faciliten a las personas, competir en orden progresivo, utilizando las habilidades más sencillas que hubiesen aprendido, hasta adquirir aquellas cada vez más complejas, que le ayudarán en alguna medida a liberarse de sus limitaciones.

El área educativa en las personas con retardo mental, se convierte en un reto, y como parte de él, la lectura cobra un papel importante.

El satisfacer esta necesidad académica básica requiere de

"Una visión ampliada...que vaya más allá de los recursos actuales, las estructuras institucionales, los planes de estudio y los sistemas tradicionales de la instrucción, tomando como base lo mejor de las prácticas en uso" (Declaración Mundial sobre Educación para todos, 1990).

Las personas con necesidades educativas especiales, no se desarrollan en forma aislada, sino dentro de un contexto familiar, social, por lo que el proceso de aprendizaje involucra estos ambientes.

En Costa Rica, la legislación vigente, reconoce sus derechos a través de la 
igualdad de oportunidades en su ley 7600 , artículo 14 en donde

"El estado garantizará el acceso oportuno a la educación a las personas independientemente de su discapacidad, desde la estimulación temprana hasta la educación superior. Esta disposición incluye tanto la educación pública como la privada en todas sus modalidades del Sistema Educativo Nacional” p. 13.

El esfuerzo debe ser conjunto, la familia debe verse como una totalidad participante dentro del sistema educativo, así como la comunidad y los diferentes profesionales que coordinen el logro de objetivos comunes.

El retardo mental se ha definido como: "un rendimiento general significativamente inferior al promedio que se relaciona o está asociado con deficiencias de la conducta adaptativa y que se manifiesta durante el período de desarrollo" (Verdugo, 1994 p. 6).

Las personas que participaron en este programa, califican dentro de esta definición, su rendimiento intelectual se sitúa al menos dos desviaciones estándar por debajo del promedio, una vez que fueron valoradas por especialistas en el campo. "La capacidad intelectual general se define por el coeficiente de inteligencia (CI o equivalente de CI) obtenido por evaluación mediante uno o más pruebas de inteligencia normalizadas $\mathrm{y}$, administrados individualmente. (p. ej., Wechsler Ingelligence Scales for ChildrenRevised, Stanford-Binet, Kaufman Assessment Battery for Children.)" (DSM IV, 1997).

\section{Objetivo}

1. Desarrollar un programa de lectura y escritura, con ocho muchachas con retardo mental, que muestren habilidades para participar en el proceso.

\section{Metodología}

Se seleccionó una muestra de doce muchachas, en forma intencional con edades entre 20 y 48 años, por lo que fue una muestra no probabilística.

Se realizó una evaluación pedagógica, para determinar los niveles de entrada de cada uno de los educandos, en donde se constató que:

a) solo tres reconocían las letras,

b) confundían las nociones de arriba abajo, derecha e izquierda, adelante atrás,

c) además, de que su vocabulario era pobre, por lo que se llevaron a cabo actividades que les permitieran ampliar su lenguaje, y que éste a su vez les facilitara la comprensión lectora.

En esta investigación, se aplicó el modelo de destrezas propuesto por Skinner (1957).

Este modelo hace énfasis en la identificación y la comprensión de la palabra, como una destreza global. Una vez identificada, se divide la palabra en sus componentes. Las acciones ejecutadas por los educandos, deben ser observables y que se puedan medir, de tal manera que durante el proceso se puedan cuantificar las conductas, hasta llegar a la automatización de las respuestas, que le permitan adquirir niveles más altos de rendimiento.

Good, (1973) recalca que para lograr una mayor efectividad en los programas, de destrezas, es necesario el planteamiento de objetivos conductuales, no descartar el desarrollo intelectual de los alumnos, además de una programación sistemática y continua, lo que permitirá una evaluación objetiva.

Como parte de este proyecto, $\mathrm{y}$ atendiendo al modelo de destrezas, se utilizaron procedimientos conductuales en donde se aplicó la teoría del reforzamiento. Se reforzaron diferencialmente las conductas de interés que se estaban trabajando.

Se utilizaron diseños de investigación intra series en donde "se pretende poner de relieve cambios de una serie de datos 
en una medida única o serie de medidas". (Mayer y Labrador, 1984, p. 131).

Dentro de estos diseños se tomó el de criterio cambiante y múltiples $\mathrm{AB}$. Se investigaron los efectos de una condición dada sobre una conducta específica. (Kazdin, 1980).

Este diseño fue propuesto por Hall y colaboradores en 1970, y se basa en el proceso de moldeamiento, que consiste en la división de una conducta en sus componentes. Cuando se utiliza este procedimiento, se refuerza cada aproximación a la conducta final deseada. (Ribes, Burckley y Walker, 1970).

En este tipo de diseño se establecen de antemano los criterios para alcanzar la conducta del sujeto, y una vez que uno de estos criterios, es alcanzado, se van aumentado los niveles de dificultad de la conducta que se desea alcanzar.

Como parte de los reforzadores se utilizaron los sociales, que son el resultado de la interacción social. Se eligieron los abrazos, el contacto físico, las alabanzas, la aprobación, las sonrisas, entre otros, por haberse determinado que eran importantes para las muchachas, los que se administraban inmediatamente después de la conducta deseada.

Paralelamente a la aplicación de los principios conductuales, se partió de las experiencias personales, como un medio para despertar el deseo de aprender a leer.

La motivación se convirtió a su vez, en una herramienta de entrada, la que se trabajó, tomando en cuenta las habilidades de las participantes, facilitando el proceso.

\section{Procedimientos}

La muestra se seleccionó en forma intencional, condición que la hace ser, una muestra no probabilística, que se caracteriza por

"el empleo del criterio de un esfuerzo deliberado para obtener muestras representativas mediante la inclusión de áreas típicas o grupos supuestamente típicos de la muestra “ (Kerlinger, 1985, p. 92).
Cobra importancia en este tipo de estudio, los conocimientos y experiencias que se tenían al respecto de la discapacidad, así como la repetición de estudios con otros sujetos.

Se definieron operacionalmente las conductas por trabajar, y se estableció una línea base para cada uno de los estudiantes, y poder así determinar el nivel operante de la conducta que se quería trabajar.

Se utilizaron procedimientos de intervención conductual, y se llevaron registros de cada sesión, para medir el avance de cada una de las muchachas.

Al inicio del programa se realizó un registro de observación. Se anotaron los cambios que iban ocurriendo.

Dentro de esta técnica de observación, se seleccionó el registro de eventos, definido por Hall (1973) como aquel en que

\footnotetext{
"un observador hace un registro acumulativo de eventos discretos de cierta clase. Este es un registro de la frecuencia de ocurrencia de eventos.... Una de las ventajas del registro de eventos es que es un procedimiento sencillo que no interfiere significativamente con las tareas rutinarias. El registro de eventos también produce un resultado numérico." P. 8 .
}

\section{Desarrollo del programa}

Inicialmente se seleccionaron doce muchachas, con quienes se trabajó con el programa el "Maestro en Casa" (diseñado por el Instituto Costarricense de Educación Radiofónica (ICER), para la alfabetización de adultos), sin embargo con el transcurrir del tiempo, hubo deserción, por causas como enfermedad o traslado a otras zonas de las muchachas. Se mantuvieron en forma constante solo ocho de ellas.

Las sesiones se llevaron a cabo semanalmente por espacio de dos años con una duración de dos horas. El programa se llevó a término con un solo instructor.

Las sesiones fueron programadas para ser trabajadas tanto en forma individual como grupal. Para el desarrollo del lenguaje oral, hubo participación de todas 
las muchachas, beneficiándose aquellas que tenían un lenguaje más pobre, en el área de comprensión y estructuración de oraciones. Esto se llevó a cabo a través de las discusiones y aportes orales, y mediante la utilización de materiales como láminas, y tarjetas entre otros. El proceso se individualizó de acuerdo a la pauta que daba cada una de las participantes, tomando en consideración su ritmo de aprendizaje, así como las dificultades específicas en el proceso tanto cognitivas como conductuales. Se tomaron en consideración otras discapacidades asociadas al retardo mental como fue la dislexia, convirtiéndose este aspecto en una dificultad más para el desarrollo del programa.

Debido a las características de la población, no fue posible trabajar con un grupo mayor de alumnos, ya que se requiere de:

a. una preparación sistemática e individualizada. Cada estudiante marcha a su propio ritmo, sus períodos de atención son muy cortos, así como su memoria a corto y largo plazo presenta dificultades, por lo que la sistematización de los programas es de vital importancia.

b. Una característica presente en las personas con retardo mental es que sus ideas son concretas y el vocabulario pobre. Debido a ello, el área de asociación y conexiones mentales debe trabajarse laboriosamente, para ayudarlos en forma eficaz en los procesos de abstracción.

Las condiciones apuntadas anteriormente, hacen que su ritmo de aprendizaje sea más lento que el resto de la población cuyas características mentales son diferentes.

Para identificar los niveles de entrada de cada una de las muchachas, se realizó una evaluación en proceso, lo que permitió determinar las habilidades de cada una de las participantes. En el área percep- tual, se trabajó la identificación de formas, memoria visual, memoria auditiva, discriminación visual, direcciones, relaciones espaciales, dominancia corporal que son requisitos para la lectura.

Para que fijaran su atención en las palabras, y se les permitiera descubrirlas con mayor facilidad se utilizaron los recursos didácticos: clave de configuración y análisis estructural, además de carteles de conversación, y de lámina palabra.

\section{Análisis de resultados}

El análisis de los datos se presenta en cuadros y gráficos. El primer cuadro muestra los resultados generales en términos porcentuales de las ocho participantes que se mantuvieron en forma constante a través del programa. Luego para efectos de presentación, se tomarán tres casos representativos, y se graficarán a partir de la línea base, para tener una idea de los avances. A través del análisis visual se pueden observar los cambios que se dieron, hasta el final de la intervención.

Los datos evidencian una variabilidad en la respuesta, demostrándose de esta manera, que se produjeron cambios en los procesos de lectura después de la intervención conductual pedagógica.

En el primer cuadro se representan, los datos generales obtenidos. En la columna de la izquierda se anotan las sílabas estudiadas desde las más simples a las más complejas, y en la columna de la derecha, en términos porcentuales, la destreza adquirida.

\begin{tabular}{ll}
\multicolumn{2}{c}{ Cuadro general } \\
\hline Sílabas estudiadas & $\begin{array}{l}\text { \% de personas } \\
\text { que obtuvieron } \\
\text { la destreza }\end{array}$ \\
\hline Vocales & $100 \%$ \\
Sílabas directas & $75 \% \quad 25 \%$ con ayuda \\
Sílaba mixta & $75 \% \quad 25 \%$ con ayuda \\
Sílaba inversa & $75 \% \quad 25 \%$ no adquirió \\
Directas consonánticas & No se han estudiado \\
Mixta consonántica & No se han estudiado \\
\hline
\end{tabular}


Cuadro No. 1

Reconocimiento de vocales

\begin{tabular}{cc}
\hline Número de persona & $\begin{array}{c}\text { Porcentaje de respuesta } \\
\text { obtenido }\end{array}$ \\
\hline N 1 & $100 \%$ \\
N 2 & $100 \%$ \\
N 3 & $100 \%$ \\
N 4 & $100 \%$ \\
N 5 & $100 \%$ \\
N 6 & $100 \%$ \\
N 7 & $80 \%$ \\
N 8 & $70 \%$ \\
\hline
\end{tabular}

Gráfico No. 1

Reconocimiento de vocales

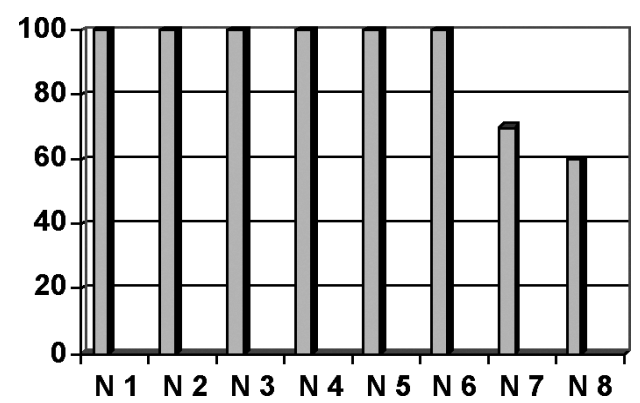

La línea vertical representa los porcentajes de respuesta alcanzados, y la línea horizontal el número de persona.

Cuadro No. 2

Reconocimiento de sílabas directas (conformadas por una consonante y una vocal)

\begin{tabular}{cc}
\hline Número de persona & $\begin{array}{c}\text { Porcentaje de respuesta } \\
\text { obtenido }\end{array}$ \\
\hline N 1 & $100 \%$ \\
N 2 & $100 \%$ \\
N 3 & $100 \%$ \\
N 4 & $100 \%$ \\
N 5 & $100 \%$ \\
N 6 & $100 \%$ \\
N 7 & $70 \%$ \\
N 8 & $60 \%$ \\
\hline
\end{tabular}

Gráfico No. 2

Reconocimiento de sílabas directas

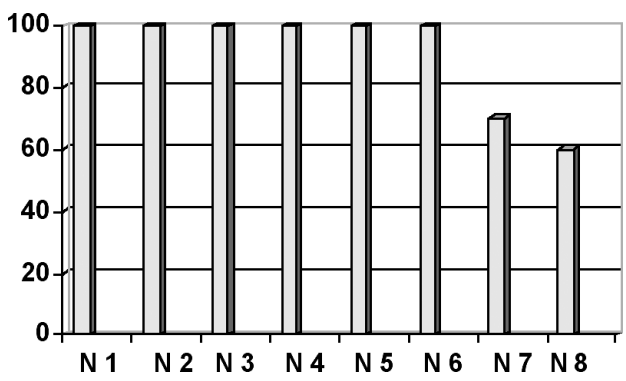

\section{Comentario}

En la persona $\mathrm{N}^{\circ} 8$ persisten los problemas de reconocimiento visual de letras, y fusión de las mismas. Se lleva a cabo el proceso con mayor dificultad que en el resto de sus compañeras, debido a la condición de dislexia asociada al retardo mental.

Cuadro No. 3

Sílabas inversas

(formada por una vocal y una consonante)

\begin{tabular}{cc}
\hline Número de persona & $\begin{array}{c}\text { Porcentaje de respuesta } \\
\text { obtenido }\end{array}$ \\
\hline N 1 & $100 \%$ \\
N 2 & $100 \%$ \\
N 3 & $100 \%$ \\
N 4 & $95 \%$ \\
N 5 & $90 \%$ \\
N 6 & $90 \%$ \\
N 7 & $60 \%$ \\
N 8 & $60 \%$ \\
\hline
\end{tabular}

Gráfico No. 3

Adquisición de sílaba inversa

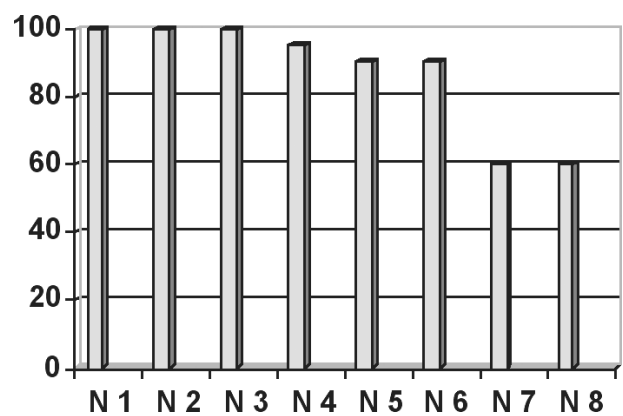




\section{Comentario}

Puede notarse el bajo nivel de rendimiento de las personas $\mathrm{N} 7$ y $\mathrm{N}$ 8. Los períodos de atención de estas personas son muy cortos, así como la memoria a corto y largo plazo, lo que repercute en su aprendizaje.

Seguidamente se representan los porcentajes obtenidos por cada una de las muchachas en la lectura de oraciones. Se anota el número de página del libro Cartilla Bribri, y la primera oración de cada una de estas páginas, además del número total de oraciones por página, para tener una idea completa de las oraciones que lee cada uno de los estudiantes.

Como puede notarse en la tabla, las personas con los números del 1 al 6 adquirieron la lectura con niveles satisfactorios.

Las personas con los números del 7 y 8 presentan mayores niveles de dificultad en la adquisición de esta destreza.

El porcentaje del $80 \%$ puede considerarse como apropiado, e interpretarse que el estudiante adquirió la habilidad para reconocer cada una de las oraciones que se presentan.

Tabla

Resumen de oraciones leídas por estudiantes

\begin{tabular}{|c|c|c|c|}
\hline Persona & Oración leída & $\mathrm{N}$ de pág. & $\%$ respuesta \\
\hline N 1 & $\begin{array}{l}\text { La familia vive en su casa. } \\
\text { En las tardes la familia se junta en la sala. } \\
\text { Rosa cuida los animales. } \\
\text { Lalo y Beto van al campo. } \\
\text { La familia tiene animales domésticos: gato, gallinas, } \\
\text { gansos, vaca, ternero, perro y algunos más. } \\
\text { La familia vive feliz en el campo. Cuántas cosas bonitas } \\
\text { tienen allí. } \\
\text { Donde vive Lalo se da el café. }\end{array}$ & $\begin{array}{l}15 \\
16 \\
17 \\
18\end{array}$ & $\begin{array}{l}100 \% \\
100 \% \\
100 \% \\
100 \% \\
100 \% \\
100 \% \\
100 \%\end{array}$ \\
\hline $\mathrm{N} 2$ & $\begin{array}{l}\text { La familia vive en su casa. } \\
\text { En las tardes la familia se junta en la sala. } \\
\text { Rosa cuida los animales. } \\
\text { Lalo y Beto van al campo. } \\
\text { La familia tiene animales domésticos: gato, gallinas, } \\
\text { gansos, vaca, ternero, perro y algunos más. } \\
\text { La familia vive feliz en el campo. Cuántas cosas bonitas } \\
\text { tienen allí. } \\
\text { Donde vive Lalo se da el café. }\end{array}$ & $\begin{array}{l}15 \\
16 \\
17 \\
18\end{array}$ & $\begin{array}{l}100 \% \\
100 \% \\
100 \% \\
100 \% \\
100 \% \\
100 \% \\
100 \%\end{array}$ \\
\hline N 3 & $\begin{array}{l}\text { La familia vive en su casa. } \\
\text { En las tardes la familia se junta en la sala. } \\
\text { Rosa cuida los animales. } \\
\text { Lalo y Beto van al campo. } \\
\text { La familia tiene animales domésticos: gato, gallinas, } \\
\text { gansos, vaca, ternero, perro y algunos más. } \\
\text { La familia vive feliz en el campo. Cuántas cosas bonitas } \\
\text { tienen allí. } \\
\text { Donde vive Lalo se da el café. }\end{array}$ & $\begin{array}{l}15 \\
16 \\
17 \\
18\end{array}$ & $\begin{array}{l}100 \% \\
100 \% \\
100 \% \\
100 \% \\
100 \% \\
100 \% \\
100 \%\end{array}$ \\
\hline
\end{tabular}




\begin{tabular}{|c|c|c|c|}
\hline Persona & Oración leída & N de pág. & $\%$ respuesta \\
\hline $\mathrm{N} 4$ & $\begin{array}{l}\text { La familia vive en su casa. } \\
\text { En las tardes la familia se junta en la sala. } \\
\text { Rosa cuida los animales. } \\
\text { Lalo y Beto van al campo. } \\
\text { La familia tiene animales domésticos: gato, gallinas, } \\
\text { gansos, vaca, ternero, perro y algunos más. } \\
\text { La familia vive feliz en el campo. Cuántas cosas bonitas } \\
\text { tienen allí. } \\
\text { Donde vive Lalo se da el café. }\end{array}$ & $\begin{array}{l}15 \\
16 \\
17 \\
18\end{array}$ & $\begin{array}{l}100 \% \\
100 \% \\
100 \% \\
100 \%\end{array}$ \\
\hline N 5 & $\begin{array}{l}\text { La familia vive en su casa. } \\
\text { En las tardes la familia se junta en la sala. } \\
\text { Rosa cuida los animales. } \\
\text { Lalo y Beto van al campo. } \\
\text { La familia tiene animales domésticos: gato, gallinas, } \\
\text { gansos, vaca, ternero, perro y algunos más. } \\
\text { La familia vive feliz en el campo. Cuántas cosas bonitas } \\
\text { tienen allí. } \\
\text { Donde vive Lalo se da el café. }\end{array}$ & $\begin{array}{l}15 \\
16 \\
17 \\
18\end{array}$ & $\begin{array}{l}100 \% \\
80 \% \\
80 \% \\
80 \% \\
80 \% \\
80 \% \\
80 \%\end{array}$ \\
\hline N 6 & $\begin{array}{l}\text { La familia vive en su casa. } \\
\text { En las tardes la familia se junta en la sala. } \\
\text { Rosa cuida los animales. } \\
\text { Lalo y Beto van al campo. } \\
\text { La familia tiene animales domésticos: gato, gallinas, } \\
\text { gansos, vaca, ternero, perro y algunos más. } \\
\text { La familia vive feliz en el campo. Cuántas cosas bonitas } \\
\text { tienen allí. } \\
\text { Donde vive Lalo se da el café. }\end{array}$ & $\begin{array}{l}15 \\
16 \\
17 \\
18\end{array}$ & $\begin{array}{l}80 \% \\
80 \% \\
80 \% \\
80 \% \\
80 \% \\
80 \% \\
80 \%\end{array}$ \\
\hline N 7 & $\begin{array}{l}\text { La familia vive en su casa. } \\
\text { En las tardes la familia se junta en la sala. } \\
\text { Rosa cuida los animales. } \\
\text { Lalo y Beto van al campo. } \\
\text { La familia tiene animales domésticos: gato, gallinas, } \\
\text { gansos, vaca, ternero, perro y algunos más. } \\
\text { La familia vive feliz en el campo. Cuántas cosas bonitas } \\
\text { tienen allí. } \\
\text { Donde vive Lalo se da el café. }\end{array}$ & $\begin{array}{l}15 \\
16 \\
17 \\
18\end{array}$ & $\begin{array}{l}80 \% \\
80 \% \\
75 \% \\
50 \% \\
50 \% \\
50 \% \\
50 \%\end{array}$ \\
\hline N 8 & $\begin{array}{l}\text { La familia vive en su casa. } \\
\text { En las tardes la familia se junta en la sala. } \\
\text { Rosa cuida los animales. } \\
\text { Lalo y Beto van al campo. } \\
\text { La familia tiene animales domésticos: gato, gallinas, } \\
\text { gansos, vaca, ternero, perro y algunos más. } \\
\text { La familia vive feliz en el campo. Cuántas cosas bonitas } \\
\text { tienen allí. } \\
\text { Donde vive Lalo se da el café. }\end{array}$ & $\begin{array}{l}15 \\
16 \\
17 \\
18\end{array}$ & $\begin{array}{l}85 \% \\
50 \% \\
50 \% \\
50 \% \\
45 \% \\
50 \% \\
50 \%\end{array}$ \\
\hline
\end{tabular}

\section{Resumen de resultados}

Para efectos de este informe se tomarán únicamente cuatro casos represen- tativos, que ilustren el proceso de adquisición de la lectura, desde la línea base, hasta el final de la intervención. 
Tabla caso No. 4

Desarrollo proceso de lectura

\begin{tabular}{|c|c|c|}
\hline Criterio & Línea base & $\begin{array}{c}\% \text { de } \\
\text { respuesta } \\
\text { obtenido }\end{array}$ \\
\hline Vocales & L.b.1 $\quad 0 \%$ & $100 \%$ \\
\hline Sílaba directa & L.b.2 $\quad 0 \%$ & $100 \%$ \\
\hline Sílaba inversa & L.b.3 $50 \%$ & $100 \%$ \\
\hline Lámina palabra & L.b.4 $70 \%$ & $100 \%$ \\
\hline Alfabeto & L.b.5 60\% & $100 \%$ \\
\hline Lectura oraciones & L.b.6 $60 \%$ & $90 \%$ \\
\hline
\end{tabular}

Gráfico caso No. 4

Desarrollo proceso de lectura

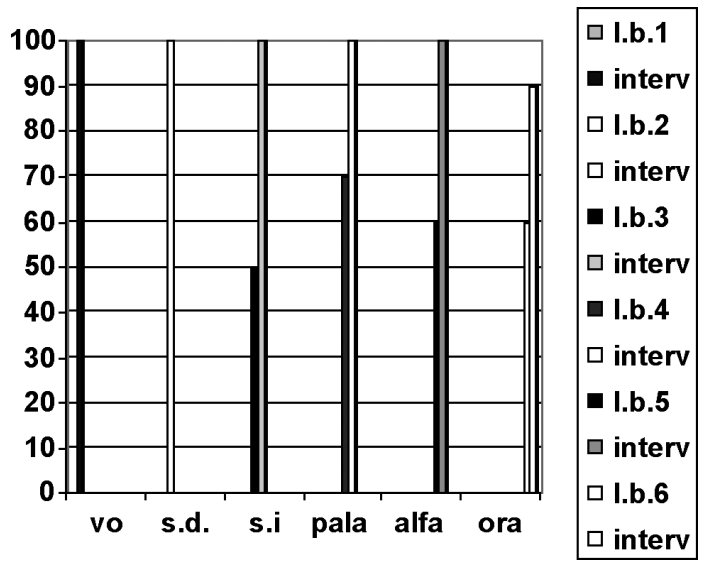

\section{Comentario}

La columna de la izquierda nos indica el nivel de cada una de las conductas sin intervención, (línea base) y la columna de la derecha, las destrezas adquiridas después de la intervención.

Como puede observarse, para cada una de las conductas se estableció una línea base que permitió determinar los niveles de entrada para las habilidades que se querían enseñar. Conforme el estudiante adquiere, la destreza, se aumenta el nivel de dificultad, y se cambian los criterios para cada una de las conductas siguientes, de tal manera que los conocimientos adquiridos, se convierten en la línea base de las etapas siguientes. El gráfico permite visualizar el avance en la lectura. En el gráfico se representa el diseño de criterios cambiantes, en donde se establecen múltiples $\mathrm{AB}$ en donde $\mathrm{A}$ está representada por la línea base y B la intervención. Conforme el estudiante adquiere la conducta, se aumenta el criterio para la siguiente etapa, lo que puede visualizarse en el gráfico.

Tabla de resultados, caso No. 5

Proceso de lectura

\begin{tabular}{|c|c|c|}
\hline Criterio & Línea base & $\begin{array}{c}\% \text { de } \\
\text { respuesta }\end{array}$ \\
\hline Vocales & L.b. $1 \quad 0 \%$ & $100 \%$ \\
\hline Sílaba directa & L.b. $220 \%$ & $100 \%$ \\
\hline Sílaba inversa & L.b. $340 \%$ & $80 \%$ \\
\hline Palabras & L.b. $450 \%$ & $90 \%$ \\
\hline Alfabeto & L.b. $560 \%$ & $100 \%$ \\
\hline Lectura & L.b. $630 \%$ & $80 \%$ \\
\hline
\end{tabular}

Gráfico caso No. 5

Desarrollo proceso de lectura

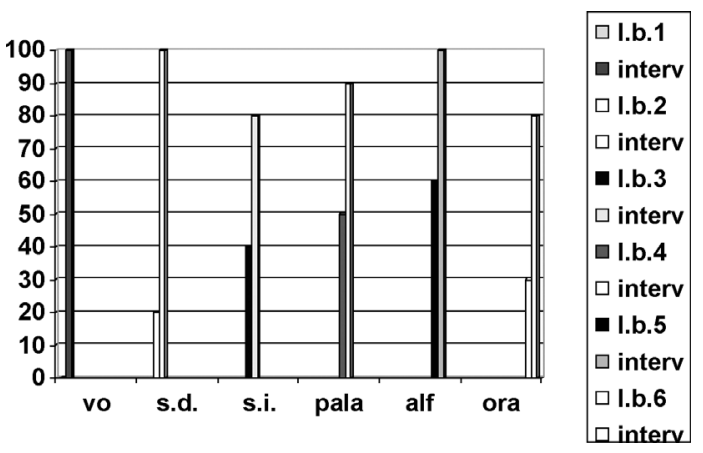

Tabla: resultados caso No. 8

Desarrollo proceso lectura

\begin{tabular}{llrc}
\hline Criterio a enseñar & Línea base & $\begin{array}{r}\% \text { de } \\
\text { respuesta } \\
\text { obtenido }\end{array}$ \\
\hline Vocales & L.b. 1 & $0 \%$ & $100 \%$ \\
Sílaba directa & L.b. 2 & $0 \%$ & $60 \%$ \\
Sílaba inversa & L.b. 3 & $0 \%$ & $50 \%$ \\
Palabra & L.b. 4 & $20 \%$ & $70 \%$ \\
Alfabeto & L.b. 5 & $20 \%$ & $50 \%$ \\
Lectura & L.b. 6 & $10 \%$ & $50 \%$ \\
\hline
\end{tabular}


Gráfico resultados caso No. 8

Proceso lectura

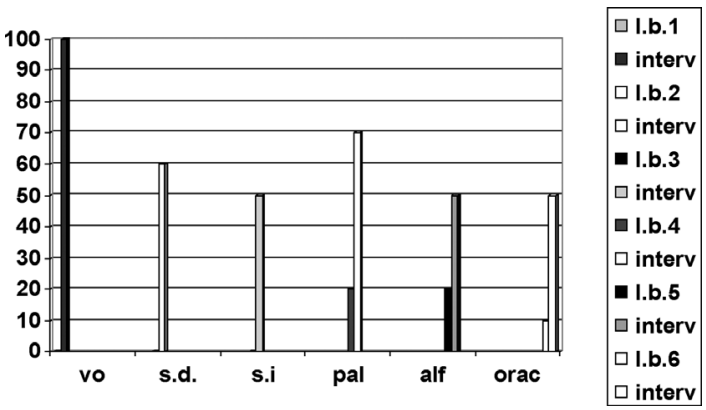

\section{Comentario}

Como puede observarse la línea base con que inició esta estudiante fue de cero, adquiriendo porcentajes de respuesta de $100 \%$ para las vocales, $50 \%$ en las sílabas directas. Una vez adquiridos estos conocimientos, la iniciación en el reconocimiento del alfabeto, de algunas palabras, así como la lectura de frases, no inició con una línea base de cero, como puede apreciarse en el gráfico, sin embargo no alcanzó los niveles deseados que nos permitan decir que adquirió la lectura en forma fluída, pero sí funcionalmente.

\section{Conclusiones}

1. Las personas adultas con retardo mental, tienen posibilidades de aprender a leer, sin embargo, los programas que con ellos se implementan no se encuentran adecuados a sus necesidades. Para llevar a cabo un buen proceso de enseñanza aprendizaje, deben ser muy bien estructurados y sistematizados, de tal manera que se les facilite la lectura.

2. Los programas para las personas con retardo mental deben sustentarse en la repetición, y apoyarse con un mayor número de ayudas audiovisuales, para facilitar la incorporación de los conocimientos, lo que ayudaría favorablemente, si tomamos en consideración los problemas en memoria a corto y largo plazo.

3. El método de lectura el maestro en casa, fue un buen apoyo para desarrollar las habilidades lectoras en muchachas con problemas en el desarrollo. Es importante hacer las adecuaciones necesarias para cada uno de los educandos, ya que no puede seguirse la guía como está dispuesta.

4. El método de lectura global que se aplicó a esta población, ayudó a su vez a la formación del lenguaje, y a la expresión oral por medio de oraciones completas. Se convirtió en una valiosa herramienta para estas personas, al enriquecer su lenguaje que era muy concreto, y aumentar en algunas de las muchachas la capacidad de abstracción. Si tomamos en consideración que las personas adultas con retardo mental tienen muy pocas oportunidades de alfabetización en el país, podríamos deducir que programas estructurados como el que se aplicó, en donde se trabajen las habilidades lectoras, les permitirían desarrollar destrezas para una mejor integración dentro del medio en que se desenvuelven.

5. El modelo de destrezas se adapta a las necesidades de las personas con problemas en el desarrollo, y permite al estudiante, a través de un análisis sistematizado avanzar a su propio ritmo, ya que divide cada una de las habilidades de lo simple a lo complejo.

6. El uso de técnicas de Terapia conductual, ayudan al incremento de aquellos repertorios deseados en los educandos, convirtiéndose en una 
herramienta muy importante para la adquisición de los conocimientos, y para la implementación de programas educativos.

\section{Referencias bibliográficas}

Condemarín, Mabel. Chadwick Neva Milicic, Madurez Escolar. Santiago de Chile, Editorial Andrés Bello. 1995.

Condemarín, Mabel. Lectura temprana. Chile. Editorial Andrés Bello, 1994.

Gray William. La enseñanza de la Lectura y de la Escritura. Suiza Organización de las Naciones Unidas para la Educación de la Ciencia y la Cultura, Talleres Atar, 1958.

Good, C.Y., Dictionary of Education, 3 ed. New York. Mac. Graw Hill Book New York Reading Association. 1993.

Hall R. El Manejo de la Conducta, el modelo de enseñanza responsive. Traducido al español por Francisco Montes. Estados Unidos. Hand H. Enterprises. In. 1973.

Kerlinger, Frederick. Investigación del comportamiento. Técnicas y Metodo- logía. México Nueva Editorial Interamericana. Edición Revisada. 1985.

Ley 7600 Igualdad de oportunidades para las personas con Discapacidad y su Reglamento. San José Costa Rica. Publicado en el Diario Oficial La Gaceta No. del 29 mayo de 1996.

Manual diagnóstico y estadístico de los trastornos mentales. DSM IV Madrid, Editorial Masson, S.A. 1997.

Mayer y Labrador Francisco. Modificación de conducta. España. Editorial Alambra. 1984.

Ministerio de Educación Pública ICER, El Maestro en Casa, San José, Costa Rica. ICER, 1993.

Shea,Thomas, Bawer Anne Marie. A. Educación Especial, un enfoque ecológico. México Editorial Mc. Graw Hill Interamericana Editores S. A. 1999.

Skinner, B.F., Verbal Behabior. New York: Appleton Century Crafts. 1957.

Verdugo, Miguel Angel. El cambio de Paradigma en la concepción de retardo Mental. España. En revista siglo cero. N 153 (25), 5, 241994.

Hannia Cabezas Pizarro Profesora, Educación Especial

Universidad de Costa Rica 
